\title{
Efectividad de las salidas frontal y lateral para la prueba de pista 300 metros CRI, patinaje de velocidad sobre ruedas
}

\author{
Effectiveness of the front and side outlets for testing CRI 300 meters track, \\ speed skating on wheels
}

\section{Eficácia das saídas frontais e laterais para testes CRI 300 metros pista, patinação de velocidade sobre rodas}

\section{Daniel A. A. Bohórquez-Páez ; Luis A.Pinzón-Castro² ;Jorge A. Obando-Bastidas ${ }^{3}$}

Licenciado en Educación Física y Deportes, MSc, PhD; Facultad de Ciencias de la Educación. Universidad de los Llanos. Licenciado en Matemáticas y Física (LMyF), MSc; Facultad de Psicología, Universidad Cooperativa de Colombia sede Villavicencio. Licenciado en Matemáticas (LM), Esp. MSc; Facultad de Contaduría Pública, Universidad Cooperativa de Colombia sede Villavicencio.

Email: daabp@hotmail.com

\section{Resumen}

Se analizó y evaluó biomecánicamente, desde el plano sagital izquierdo, la Salida Frontal (SF) y la Salida Lateral (SL) en pruebas de velocidad de pista Contra el Reloj Individual (CRI), de once (11) Patinadores de Velocidad Sobre Ruedas (VSR), empleando el Software SIMI ${ }^{\circ}$ Twinner Pro y el software SPSS (versión 19); lo que permitió determinar que la SF es la más efectiva que la SL para el desarrollo de las pruebas CRI en el patinaje de VSR.

Palabras clave: patinaje de velocidad sobre ruedas, pruebas de velocidad Contra el Reloj Individual, salida frontal, salida lateral, trocánter izquierdo.

\begin{abstract}
We analyzed and evaluated biomechanically, from the left sagittal plane, the front output (SF) and the side outlet (SL) in speed track individual time trial (CRI) of eleven (11) skaters penultimate (RSV) using the software SIMI ${ }^{\circ}$ Twinner Pro software and SPSS (version 19); which it allowed to determine that the SF is more effective than the SL for the development of tests CRI RSV skating.
\end{abstract}

Key words: in-line speeds kating, individual time trial raced against the clock, front start, side start, left trochanter (upper hip). 


\section{Resumo}

Foram analisados e avaliados biomecanicamente, a partir do plano sagital esquerdo, a saída frontal (SF) ea saída lateral (SL) na velocidade rastrear contra-relógio individual (CRI) de onze (11) patinadores penúltimo (RSV) usando o software SIMI ${ }^{\circ}$ Twinner software Pro e SPSS (versão 19); que permitiu determinar que o SF é mais eficaz do que o SL para o desenvolvimento de testes de CRI RSV patinagem.

Palavras-chave: Roda de patinação de velocidade, testes de velocidade Contra Relógio Individual, saída da frente, saída lateral, trocânteresquerdo.

\section{Introducción}

El patinaje de VSR o patinaje de velocidad en línea, es una de las modalidades de más rápido desarrollo en el patinaje competitivo mundial. Dentro de las distintas modalidades del patinaje de velocidad, se desarrollan las pruebas CRI de los 300 metros (m) pista y los 200 m Ruta. Dichas pruebas, se han Ilevado a cabo por más de 40 años; y en el patinaje VSR se realizan con Salida Frontal (SF). A través de ésta se han mejorado marcas y se han impuesto récords mundiales, hasta el Mundial de patinaje celebrado en Gijón-España 2008. Según datos de la Real Federación Española de Patinaje, se batió "...el record de los 300 metros gracias a la brillante actuación de la americana B. Bowe, que dejó la marca en 26,611 segundos (s), realizando una Salida Lateral $(\mathrm{SL})^{\prime \prime}$. El récord estaba en poder de la italiana Nicoletta Falcone, quien con una SF impuso en Cali-Colombia, en el año 2007, el record mundial con 26,971 (s). En la actualidad, el récord del mundo femenino de los $300 \mathrm{~m}$, lo ostenta la colombiana Jercy Puello Ortiz, logrado en la final del Campeonato del Mundo de Ostende-Bélgica 2013. Empleando una SF, tal como lo corrobora la Federación Internacional de Roller Sports, (FIRS) al resaltar el nuevo récord mundial y el registro de 25,993 (s).

La SF y la SL difieren básicamente en la ubicación de los patines, en la línea de salida y con respecto a la dirección de la competencia; siendo la salida según De Koning, et al, (1989) una de las partes más importantes de la carrera. Lo cual significa que casi el $80 \%$ de la variación en los tiempos finales, se asocia con la medida en que el patinador es capaz de acelerar durante los primeros $100 \mathrm{~m}$.

Teniendo en cuenta que la salida en el patinaje de VSR según Rosas, et al, (2006) es “...una transición abrupta del estado estático, en que se encuentra el patinador, a una fase dinámica en la que se busca una rápida aceleración que permita posteriormente la realización de otras técnicas inmersas dentro de la prueba". Permitiendo al patinador, según Acero, et al, (2003), "...adquirir la velocidad y por ende la aceleración necesaria que, según la técnica que emplee, pueda retardarse o acelerarse y así perder o ganar, un tiempo valioso para el total de la prueba."

Se empleó el Software SIMI ${ }^{\circ}$ Twinner Pro, con el cual se obtuvo datos de las variables (tiempo, distancia, velocidad, aceleración, distancia total promedio de la 1 ra zancada Homolateral, distancia total promedio desde la línea de salida a el 1er paso, distancia total promedio del 1 er paso, distancia total promedio de la 1ra zancada Contralateral, distancia total promedio del 2 do paso, distancia total promedio alcanzada en la pista activa por el patín), para determinar la efectividad de la SF y la SL para el desarrollo de la prueba de Pista $300 \mathrm{~m} \mathrm{CRI}$, mediante el software SPSS versión 19. Lo anterior con el propósito de dar cumplimiento al objetivo de esta investigación, que es determinar la efectividad de la SF y la SL para la prueba de Pista 300 m CRI, en el patinaje de VSR. 


\section{Materiales y métodos}

Esta investigación fue llevada a cabo con once (11) Patinadores de VSR, en perfecto estado de salud.

\section{- Materiales}

Vídeo-cámara JVC Everio GZ-MG130U, cinta de marcado, conos de $25 \mathrm{~cm}$, cinta de enmascarar, trípode, pie de rey, pista de patinaje de VSR, ordenador portátil, software Ulead Video Studio10 plus, plantillas de Excel 2007, software SIMI ${ }^{\circ}$ Twinner Pro y software SPSS versión 19.

\section{- Método}

\section{Tipo de muestreo:}

No probabilístico intencional de Casal, J. y Mateu, E. (2003). La población participante en la presente investigación son los patinadores de VSR de Villavicencio, Meta-Colombia, todos ellos, ubicados en el TOP 10 del ranking nacional de Colombia. La muestra seleccionada para desarrollar la presente investigación fue de once (11) Patinadores de VSR. Cuatro hombres quienes representan el $36,4 \%$ y siete mujeres quienes representan el $63,6 \%$.

\section{Desarrollo metodológico:}

En previos se conoció que los patinadores expertos se encontraban en perfecto estado de salud. Fueron informados junto a sus representantes legales, dado que los patinadores expertos son menores de edad. Según la ley 27 de 1977, Artículo 1ero encontramos que:

"Para todos los efectos legales, Ilámese mayor de edad o simplemente mayor, a quien ha cumplido diez y ocho (18) años".

Los patinadores se encuentran entre los 11 y los 14 años, por esto, aclaradas las dudas que surgieron para su participación, fueron autorizados mediante el Consentimiento Informado. Adicional fue autorizada la utilización de imágenes de ellos en publicaciones científicas y con la reserva científica necesaria.

Esta investigación valoró biomecánicamente, desde el plano sagital izquierdo, la SF y la SL, utilizadas para el desarrollo de la prueba de velocidad, en Pista de $300 \mathrm{~m} \mathrm{CRI} \mathrm{en} \mathrm{patinaje}$ de VSR de patinadores expertos. Se utilizó el software SIMI $^{\circ}$ Twinner Pro para obtener resultados de las variables de la investigación y el Software SPSS versión 19.

La toma de videos digitales se hizo con cámaras compatibles de velocidad normal y alta frecuencia. Específicamente se utilizó la videocámara JVCeverioGZ-MG130U, ubicada en el centro de la trayectoria del movimiento a una altura de $102 \mathrm{~cm}$ de la superficie de la pista, sobre un trípode y con una distancia cámara-patinador de $15 \mathrm{~m}$. La filmación de los movimientos objeto de estudio fue en condiciones de luz natural a las 11:30 a.m. La distancia real fue de $460 \mathrm{~cm}$; la medida fílmica fue de $17,9 \mathrm{~cm}$; con un factor de conversión de 25,698 cm.

Sobre los patinadores expertos se ubicaron 16 puntos de referencia, a saber, 12 puntos anatómicos:

2 Acromial derecho e izquierdo (D/I)

2 Trocantéreo (D/I)

2 Tibial-Medial (D/I)

2 Tibial-Lateral (D/I)

2 Maléolar-Tibial (D/I)

2 Maléolar-Peroneal

Y 4 puntos de referencia en el implemento: 2, primera rueda del patín (D/I); y 2, última rueda del patín (D/I); con referencia en el enunciado "SC-14" de Dempster, W (1955).

Se hicieron seis (6) tomas videográficas, tres de la SF y tres de la SL. Se pasaron las tomas 
videográficas al ordenador mediante el IEEE 1394 Firewire; posteriormente, se analizaron los videos y se escogió el intento de mayor velocidad (del TI) de la SF y la SL, de los tres realizados, por cada uno de los patinadores expertos participantes en la investigación.

Después se hizo la clasificación y división de los campos visuales $30 \mathrm{c} / \mathrm{s}$ que marcaban el inicio y el final de cada una de las cuatro fases de la SF y la SL, para obtener los kinegramas que marcan el inicio y el fin de cada una de las cuatro fases del movimiento evaluado, para comprensión, recolección y orden de los datos cuantitativos. Inmediatamente, mediante el SIMI ${ }^{\circ} \mathrm{Twinner}$ Pro se recolectaron los datos de cada uno de los patinadores y de cada una de las variables propuestas, para el desarrollo de esta investigación, los cuales fueron organizados en Excel.

Posterior a esto, se empleó el software SPSS versión 19 para hacer el análisis de datos a los cuales se les aplicó la prueba de normalidad de Shapiro-Wilk y la prueba de diferencia de promedios al 95\% de confianza, para obtener resultados, desarrollar la discusión de los mismos y plantear las conclusiones.

\section{- Resultados estadísticos de las variables de la investigación}

Tabla 1. Estadística de las variables de la investigación, SF y SL

\begin{tabular}{|c|c|c|c|c|c|}
\hline & VARIABLES & SF & SL & $\mathbf{F}$ & P VALUE \\
\hline \multirow{5}{*}{ 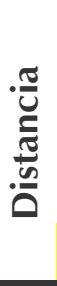 } & FASE 1 & $0,57 \pm 12 \%$ & $0,32 \pm 25,6 \%$ & $68,33^{*}$ & 0 \\
\hline & FASE 2 & $0,45 \pm 30,5 \%$ & $0,25 \pm 34 \%$ & $15,55^{*}$ & 0 \\
\hline & FASE 3 & $0,39 \pm 32,7 \%$ & $0,37 \pm 29,9 \%$ & $0,16^{*}$ & 0,68 \\
\hline & FASE 4 & $0,53 \pm 21,1 \%$ & $0,41 \pm 24,5 \%$ & $6,46^{*}$ & 0,01 \\
\hline & TOTAL & $1,97 \pm 9,8 \%$ & $1,36 \pm 9,6 \%$ & $74,04 *$ & 0 \\
\hline \multirow{5}{*}{$\begin{array}{l}\mathscr{O} \\
\frac{2}{E} \\
\frac{E}{0} \\
i=\end{array}$} & FASE 1 & $0,33 \pm 12,3 \%$ & $0,30 \pm 20 \%$ & $1,53^{*}$ & 0,23 \\
\hline & FASE 2 & $0,16 \pm 26,4 \%$ & $0,13 \pm 37 \%$ & $2,93^{*}$ & 0,1 \\
\hline & FASE 3 & $0,14 \pm 30,7 \%$ & $0,19 \pm 32,3 \%$ & $101,00 * *$ & 0,08 \\
\hline & FASE 4 & $0,16 \pm 21,9 \%$ & $0,14 \pm 21,3 \%$ & $103,00 * *$ & 0,1 \\
\hline & TOTAL & $0,80 \pm 6,4 \%$ & $0,76 \pm 11,2 \%$ & $110,50^{* *}$ & 0,28 \\
\hline \multirow{5}{*}{$\begin{array}{l}\frac{0}{0} \\
\frac{\pi}{U} \\
\frac{0}{0} \\
\frac{0}{j}\end{array}$} & FASE 1 & $1,82 \pm 11,83 \%$ & $1,08 \pm 25 \%$ & $49,725^{*}$ & 0 \\
\hline & FASE 2 & $3,11 \pm 4,69 \%$ & $1,64 \pm 27,27 \%$ & $106,24 *$ & 0 \\
\hline & FASE 3 & $2,32 \pm 40,95 \%$ & $1,99 \pm 17,53 \%$ & $1,19^{*}$ & 0,28 \\
\hline & FASE 4 & $3,24 \pm 14,12 \%$ & $2,94 \pm 14,25 \%$ & $2,63^{*}$ & 0,12 \\
\hline & TOTAL & $2,44 \pm 10,14 \%$ & $1,79 \pm 8,87 \%$ & $1,98^{*}$ & 0,17 \\
\hline \multicolumn{2}{|c|}{ Aceleración TOTAL } & $3,38 \pm 19 \%$ & $2,73 \pm 12 \%$ & $74,08^{*}$ & 0 \\
\hline \multicolumn{2}{|c|}{ DIST 1A ZAN HO } & $1,32 \pm 12,32 \%$ & $0,74 \pm 47,71 \%$ & $67,50^{* *}$ & 0 \\
\hline \multicolumn{2}{|c|}{ DIST LIINEA S } & $0,60 \pm 29,38 \%$ & $0,29 \pm 41,30 \%$ & $22,92^{*}$ & 0 \\
\hline \multicolumn{2}{|c|}{ DIS 1ER PASO } & $0,70 \pm 14,50 \%$ & $0,37 \pm 30,11 \%$ & $51,23^{*}$ & 0 \\
\hline \multicolumn{2}{|c|}{ DIS 1 ZAN CONT } & $1,26 \pm 25,63 \%$ & $0,93 \pm 10,53 \%$ & $10,66^{*}$ & 0 \\
\hline \multicolumn{2}{|c|}{ DIST 2 PASO } & $0,65 \pm 33,72 \%$ & $0,63 \pm 14,94 \%$ & $0,08^{*}$ & 0,77 \\
\hline \multicolumn{2}{|c|}{ DIST PISTA ACT } & $1,78 \pm 10,47 \%$ & $1,41 \pm 10,37 \%$ & $27,46^{*}$ & 0 \\
\hline
\end{tabular}


En la tabla 1, se observan los resultados estadísticos de las variables de la investigación, que posteriormente serán analizados y empleados como fundamentos en las conclusiones. Para una lectura más expedita de la tabla, procédase a interpretar mediante las siguientes abreviaturas:

DIST 1A ZAN HO: Distancia (m) total promedio de la 1 ra zancada Homolateral.

DIST LÍNEA S: $\quad$ Distancia total promedio desde la línea de salida a el 1 er paso.

DIS 1ER PASO: $\quad$ Distancia $(\mathrm{m})$ total promedio del 1 er paso.

DIS 1 ZAN CONT: Distancia $(\mathrm{m})$ total promedio de la 1 ra zancada Contralateral.

DIST 2 PASO: $\quad$ Distancia $(m)$ total promedio del 2 do paso.

DIST PISTA ACT: $\quad$ Distancia $(m)$ total promedio alcanzada en la pista activa por el patín.

\section{- Análisis estadístico}

Para determinar la efectividad de la SF y la SL para la prueba 300 m Pista CRI en patinaje de VSR se analizaron los resultados de las variables Velocidad Total promedio (del TI), Aceleración (del TI), y Distancia total promedio alcanzada en la pista activa por el patín, con la prueba de diferencia de promedios al 95\% de confianza.

Tabla 2. Velocidad Total promedio (del TI), Aceleración (del TI), y Distancia total promedio alcanzada en la pista por el patín, de la SF y la SL.

\begin{tabular}{|c|c|c|c|c|c|c|c|}
\hline & & PROM & P VAL & IE NO & MALI & DAD & \\
\hline & & SF & SL & SF & SL & PRUEBA & P VALUE \\
\hline & FASE 1 & $1,82 \pm 11,18 \%$ & $1,08 \pm 25,18 \%$ & 0,52 & 0,89 & ANOVA & 0 \\
\hline & FASE 2 & $3,11 \pm 4,69 \%$ & $1,64 \pm 27,27 \%$ & 0,02 & 0,01 & ANOVA & 0 \\
\hline$\frac{\pi}{\frac{\pi}{2}}$ & FASE 3 & $2,32 \pm 40,95 \%$ & $1,99 \pm 17,53 \%$ & 0,04 & 0,24 & $\begin{array}{c}\text { W de } \\
\text { Wilcoxon }\end{array}$ & 0,87 \\
\hline$\sum^{\bar{\nu}}$ & FASE 4 & $3,24 \pm 14,12 \%$ & $2,94 \pm 14,25 \%$ & 0,77 & 0,34 & ANOVA & 0,12 \\
\hline & TOTAL & $2,44 \pm 10,14 \%$ & $1,79 \pm 8,87 \%$ & 0,04 & 0,32 & $\begin{array}{c}\text { W de } \\
\text { Wilcoxon }\end{array}$ & 0 \\
\hline Acele & ción TOTAL & $3,38 \pm 19 \%$ & $2,51 \pm 12 \%$ & 0,97 & 0,02 & $\begin{array}{c}\text { W de } \\
\text { Wilcoxon }\end{array}$ & 0 \\
\hline & ACTIVA & $1,78 \pm 10,47 \%$ & $1,41 \pm 10,37 \%$ & 0,69 & 0,27 & ANOVA & 0 \\
\hline
\end{tabular}

En la tabla 2, figura 1, 2 y 3, se evidencia que la velocidad total promedio (del TI) (SF: $2,44 \pm 10,14 \%$ y SL: $1,79 \pm 8,87 \%$ ) el ( $p$ value $=0,00$ ), presenta una diferencia significativa de la velocidad total promedio (del TI) a favor de la SF sobre la SL. En cuanto a la aceleración total

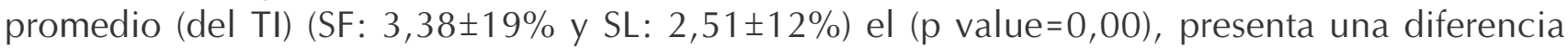
significativa de la aceleración total promedio (del TI) a favor de la SF sobre la SL. Respecto a la Distancia total promedio alcanzada en la pista activa (DIST ACTIVA) por el patín en Pre-Test (SF: $1,78 \pm 10,47 \%$ y SL: $1,41 \pm 10,37 \%$ ), el ( $p$ value $=0,00)$ presenta una diferencia significativa de la Distancia total promedio alcanzada en la pista activa por el patín, a favor de la SF sobre la SL. 


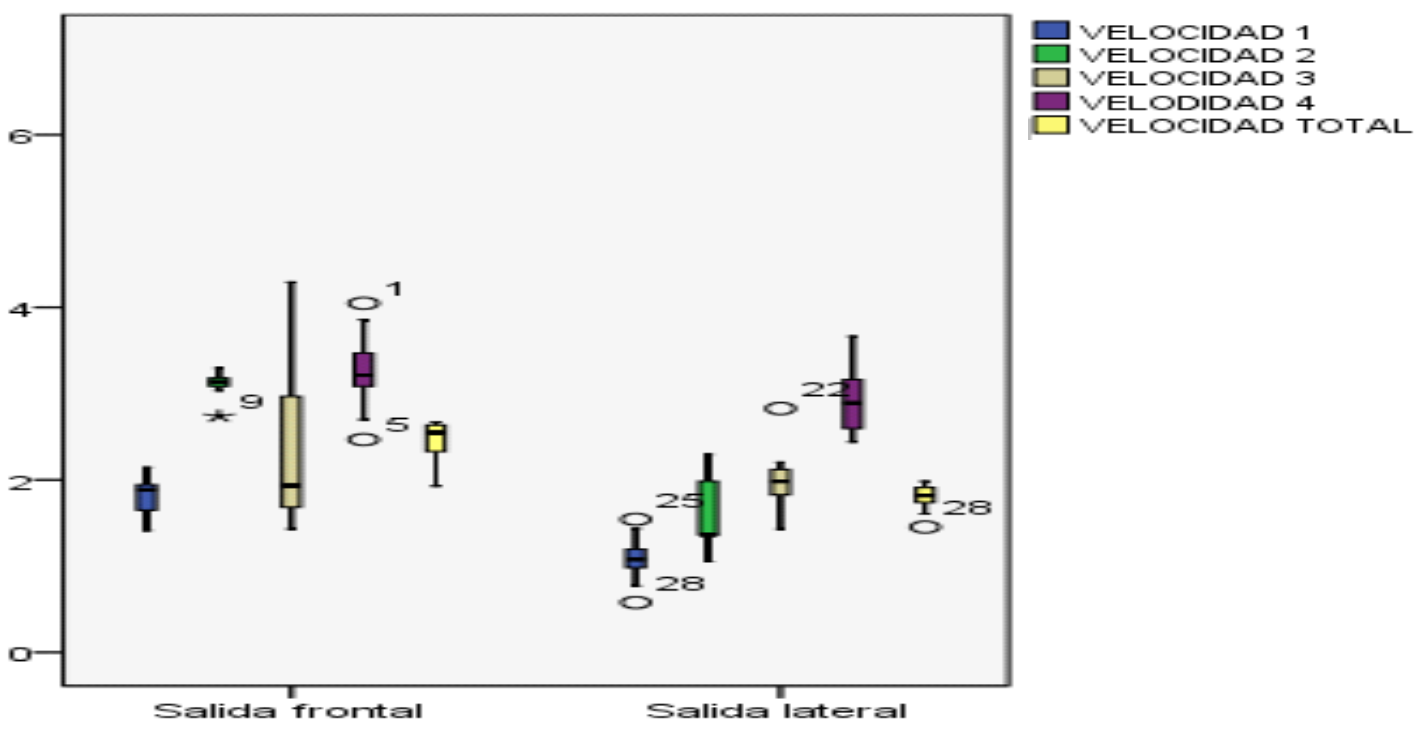

Figura 1. Velocidad (del TI) SF y SL.

En la figura 1, se evidencia que la velocidad total promedio (del TI), presenta una diferencia significativa a favor de la SF sobre la SL.

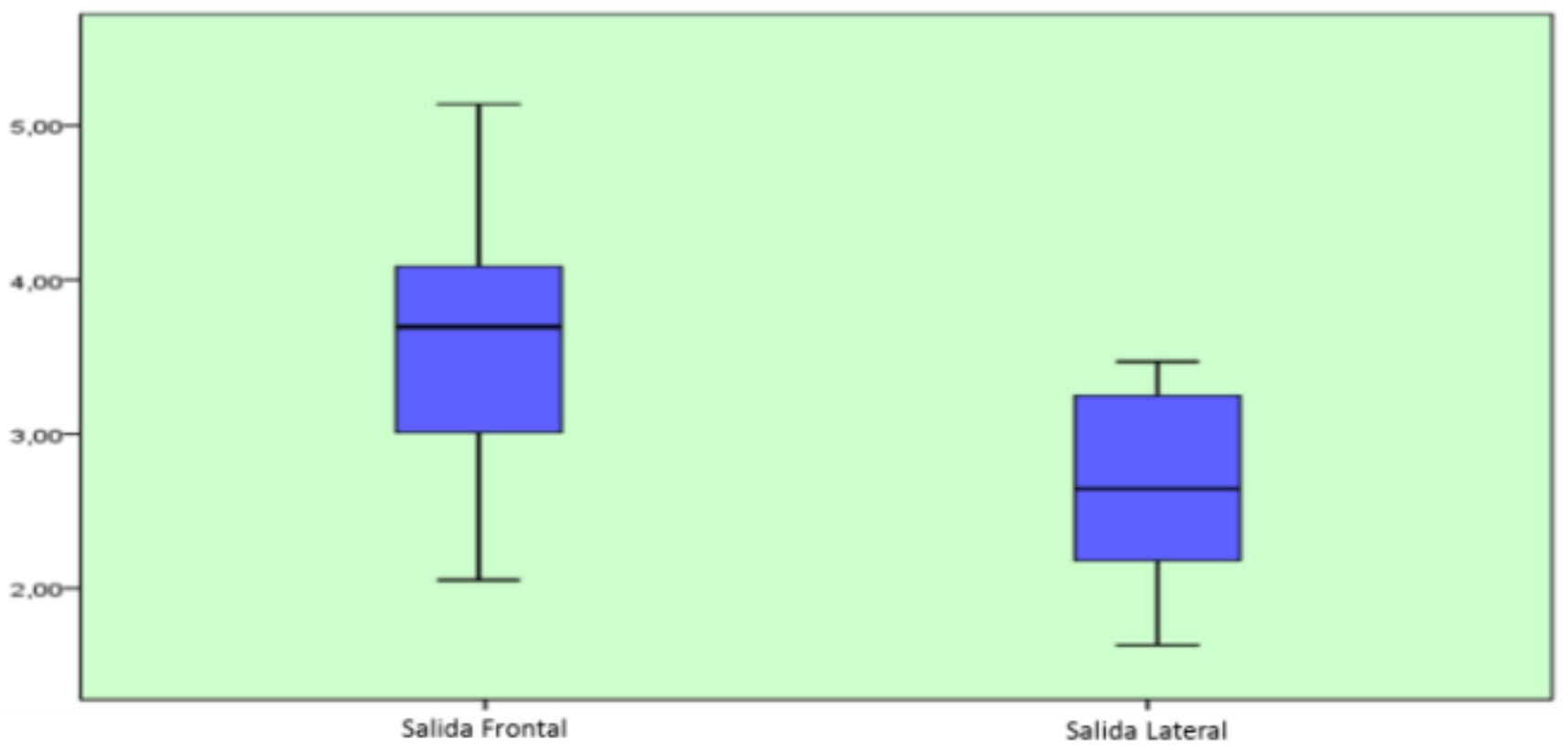

Figura 2. Aceleración (del TI) SF y SL.

En la figura 2, se observa que la aceleración total promedio (del TI), presenta una diferencia significativa a favor de la SF sobre la SL. 


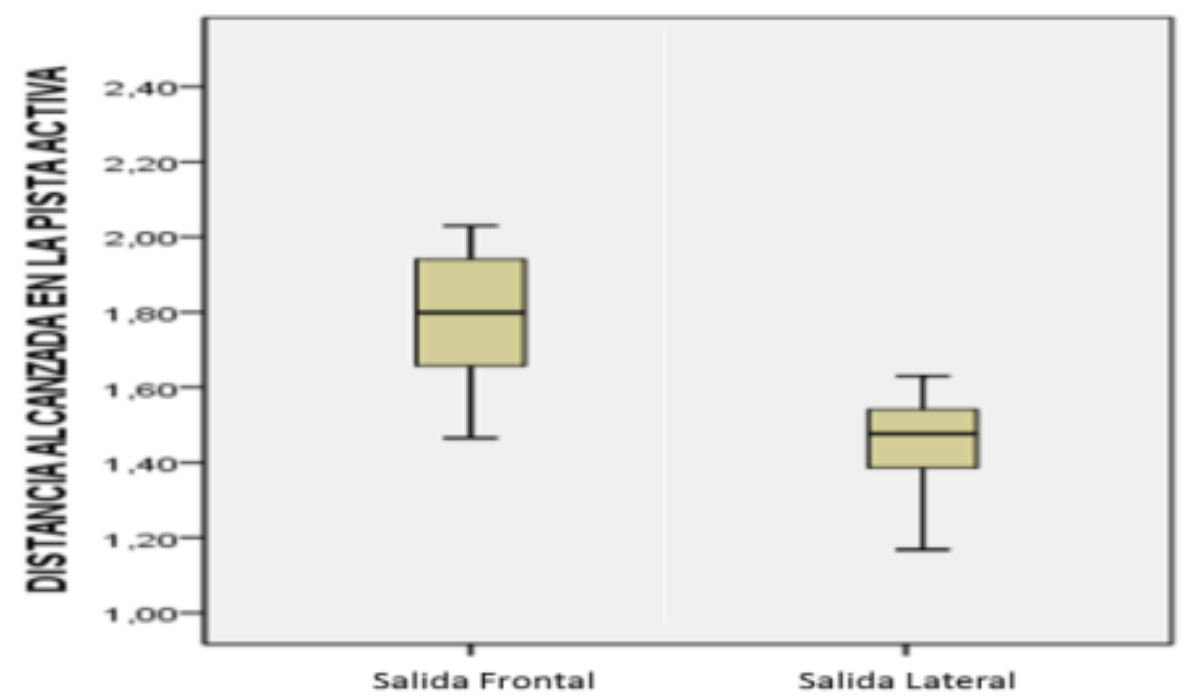

Figura 3. Distancia total promedio alcanzada en la pista activa por el patín con SF y SL.

La figura 3 presenta con respecto a la (DIST ACTIVA) Distancia total promedio alcanzada en la pista activa por el patín, una diferencia significativa a favor de la SF sobre la SL. En términos de la prueba de hipótesis, se aprueba la hipótesis nula y se rechaza la hipótesis alternativa, lo cual hace aceptar que la SF produce resultados que son efectivos para obtener mayor velocidad total promedio (del $\mathrm{TI})$, mayor aceleración total promedio (del $\mathrm{TI}$ ) y mayor distancia total promedio alcanzada en la pista activa por el patín, para las pruebas CRI en patinaje de VSR.

\section{Discusión}

Se debe resaltar que existen marcados limitantes para llevar a cabo este apartado, debido a los escasos referentes sobre la "SF en el patinaje de VSR" y los nulos referentes sobre "SL en el patinaje de VSR"; por lo cual, debido a la similitud y la gran cantidad de investigaciones emprendidas sobre patinaje de velocidad en hielo y Hockey, se emplearán algunas de éstas, para desarrollar la discusión de los resultados obtenidos con esta investigación.

La salida, según De Koning J. et al. (1989), es una de las partes más importantes de la carrera, debido a que casi el $80 \%$ de la variación en los tiempos finales se asocia con la medida en que el patinador es capaz de acelerar durante los primeros $100 \mathrm{~m}$. De Koning J. et al. (1992) también afirman que hay una notable correlación entre la aceleración del patinador en el primer segundo de la carrera (salida) y el tiempo final.
Con respecto al rendimiento del patinaje de velocidad, de acuerdo con De Koning (Ibíd), está determinado por la capacidad que tiene el patinador de producir energía externa, la cual es necesaria para superar la fricción del aire y el hielo, ambos dependientes de la velocidad. Contrario a lo planteado por Noordhof D. et al. (2013), quienes establecieron que la disminución en la velocidad no era debido a la fricción del aire, ni a modificaciones de la técnica -como los ángulos de la rodilla y el ángulo del tronco- sino que puede ser parcialmente atribuida a la disminución de la eficacia. A lo que De Koning J et al. (1992), denomina energía anaeróbica, la que los patinadores parecen ser capaces de liberar considerablemente, al inicio de la carrera (salida) con respecto a los patinadores de nivel de rendimiento inferior. Similar a lo que plantea Noordhof D. et al. (2013), al afirmar que la disminución en la producción de energía se asocia con la fatiga.

Los componentes cualitativos de la SF en el 
Patinaje de VSR se cimientan en las siguientes argumentaciones:

Publow, B. (1999) describe en cinco pasos, los aspectos más importantes de la SF sin determinar nombres. Ni para las fases, ni los elementos espaciotemporales de cada paso. Marcelloni P. (2004) señala seis fases, teniendo en cuenta que el cuerpo se mueve antes de mover el patín, para activar la célula de movimiento. Ergo, se considera que estas dos descripciones no son muy precisas, por cuanto las características para desarrollar el análisis, se pueden volver subjetivas, al no precisarse claramente cuándo termina cada fase y por no manejar un nombre en cada fase o paso. Por consiguiente los componentes descriptivos no son concretos.

Acero, J. et al, (2003) valoraron cinemáticamente desde el plano sagital la SF de un patinador de carreras, mediante un estudio piloto en (2D), empleando el método de Acero J.et al. (2001), donde describen siete fases que permiten determinar que la importancia de los pasos y la buena ejecución de la salida, radica en gran porcentaje en la adquisición temprana o tardía de la velocidad y por ende, de la aceleración inicial de la prueba. En cuanto a Rosas S. et al. (2006) mediante una observación directa del evento y de la actividad articular existente, generan cuatro fases determinantes para la búsqueda de mayor aceleración en la SF. Mantilla E. (2006), señala una descripción de la SF en el Patinaje de carreras basada en la observación directa del evento. Durante los años que él se ha desempeñado como entrenador de este deporte y los puntos de vista de los investigadores, se debe resaltar que, como está enunciado y evidenciado en el desarrollo de esta investigación, la descripción más pertinente en el ámbito deportivo y científico, es la establecida por Rosas S. et al., (2006), por cuanto permite una fácil comprensión para cualquier lector.

Seguidamente, se presenta la discusión de los resultados cuantitativos. Haciendo hincapié una vez más de las pocas referencias de investigaciones de la SF en el Patinaje de VSR y la no existencia de referentes teóricos sobre la SL en el Patinaje de VSR.

Acero J. et al. (2003), en el estudio piloto en (2D) concluyeron que el tiempo total empleado en la SF fue de 10.263 s. Siendo la fase 7, donde se empleó menos tiempo $(0.264 \mathrm{~s})$ y la de mayor durabilidad la fase 2 (3.762s). En cuanto a la velocidad lineal $(\mathrm{m} / \mathrm{s})$ de la cadera, rodilla y tobillo derecho, se estableció que el tobillo en la fase 6 y 7 tuvo, una mayor contribución en la adquisición de la velocidad lineal; que el comportamiento de la velocidad angular promedio $(\% / \mathrm{s})$ y relativa a la horizontal, es mayor en este caso en el muslo izquierdo pues es el hemisferio que da el primer paso y responsable inicial de la consecución de la aceleración corporal. Pero, todo lo anterior, se vuelve un estudio de caso, lo cual permite pensar como McMillan J. y Schumacher S. (1993) cuando advierten que la muestra no es representativa de una población. Adicional a ello, los resultados no son claros ni de fácil interpretación y comprensión.

En la caracterización cinemática hecha por Rosas S. et al. (2009), de la SF en el Patinaje de VSR y los resultados obtenidos por ellos, se pueden aceptar y darle confiabilidad a los datos, resultados y análisis de los resultados de la presente investigación. Debido a que tal, como lo plantea (Mann y Herman, citados por Aron M. et al. (2006), está demostrado que la alta velocidad horizontal -en el caso de esta investigación- el punto de referencia (del TI), tiene una buena correlación con un buen rendimiento en el sprint.

\section{Conclusión}

La salida más efectiva para realizar la prueba de 300 m Pista CRI en el patinaje de VSR, es la SALIDA FRONTAL. 


\section{Agradecimientos}

Al grupo de los once (11) Patinadores de Velocidad Sobre Ruedas (VSR); al entrenador del proceso IDERMETA Edgar Mantilla Moreno. A la Liga de Patinaje del Meta y a Sandra Marcela Rosas Rodríguez.

\section{Referencias}

Acero J, Ibargüen H, Lozano B. 2001. El fenómeno del Déficit Bilateral (DBL) en deportistas: progresos 1. Memorias Simposio Ciencias Aplicadas al Deporte en el Valle del Cauca. Cali. Colombia. Editorial Kinesis. 75-84 pp.

Acero J, Palomino A, Ibarguen H, Carmona C. (2003). Valoración Cinemática (2d) Sagital de la Salida de un Patinador de Carreras: Un Estudio Piloto. Instituto de Investigaciones \& Soluciones Biomecánicas. CaliColombia. Magacín Spagatta.

Aron M, Aaron L, Robert C. 2006. Determinantes Cinemáticos de la Aceleración Temprana en Atletas que Practican Deportes de Campo. Publice Standard. Pid: 594.

Casal J, Mateu E. Tipos de muestreo. Revista Epidem. Med. Prev. 2003; (1): 3-7.

De Koning J, De Groot G, Van Ingen Schenau G. Mechanical Aspects of the Sprint Start in Olympic Speed Skating. International Journal Of Sport Biomechanics. 1989; 5(2):151-168.

De Koning J, De Groot G, Van Ingen Schenau G. A Power Equation For The Sprint In Speed Skating. International Journal Of Sport Biomechanics. 1992; 25(6):573-580. 0021-9290/92. Impreso en Gran Bretaña. Pergamon Press Ltd.

Federación Internacional de Roller Sports. World Speed Records. Recuperado en:
(http://www.rollersports.org/RollerSports/s peed/record.html).

Mantilla E. 2006. Patinaje de carreras, técnicas del patinaje sobre ruedas, patín en línea. Cali: Editorial Kinesis.

Marcelloni P. 2004. Curso de Técnico Superior en Patinaje de Velocidad. Apuntes de Técnica del Patinaje de Velocidad. Madrid-España.

McMillan J, Schumacher S. 1993. Research in education: A conceptual introduction. (Tercera edición). New York: Harper Collins College Publishers.

Noordhof D, Foster C, Hoozemans M, De Koning J. Changes in Speed Skating Velocity in Relation to Push-Off Effectiveness. International Journal of Sports Physioiogy and Performance. 2013; 8:188194. Human Kinetics, inc.

Publow B. 1999. Speed on SkatesEd. Canada: Human Kinetics.

Real Federación Española de Patinaje. Reportajes: Patinaje de velocidad. Gijón bate records. Recuperado de Rosas S, Contreras D, Rojas D. Descripción cualitativa y cuantitativa de las cuatro primeras zancadas de la salida frontal en la prueba de 300 metros C.R.I. en patinaje de carreras. (Primer acercamiento), CLON: Revista de la facultad de Salud. 2006; 4(2):52-59. Universidad de Pamplona. Pamplona-Colombia.

Rosas S, Contreras D, Rojas D. Caracterización cinemática (plano sagital) de las cuatro primeras zancadas de la salida frontal en la prueba de $300 \mathrm{mtsC}$.R.I. en patinaje de carreras (segundo acercamiento).Ímpetus. Educación física, recreación y deporte. Universidad de los Llanos.Programa Licenciatura en Educación Física y Deportes. 2009; 3(4): 26-31. 